\title{
Defect minimized Ag-ZnO microneedles for photocatalysis
}

\author{
Sanjay Gopal Ullattil ${ }^{1}$ (D) M. J. Jabeen Fatima ${ }^{2} \cdot$ Ahmed Abdel-Wahab $^{1}$
}

Received: 30 October 2019 / Accepted: 25 May 2020 / Published online: 23 June 2020

(C) The Author(s) 2020

\begin{abstract}
A facile solution processing strategy has been developed for the formation of Ag-modified $\mathrm{ZnO}$ microneedles at various calcination temperatures such as 300,500 , and $700{ }^{\circ} \mathrm{C}$ (AZ3, AZ5, and AZ7 respectively). Due to the heavy doping of $\mathrm{AgNO}_{3}, \mathrm{Ag}^{+}$ions have been incorporated in to the crystal lattice of $\mathrm{ZnO}$ in all the Ag- $\mathrm{ZnO}$ samples, which facilitated the formation of $\mathrm{Ag}-\mathrm{ZnO}$ microneedle morphology with minimized defect states, and obviously, the plasmon peaks were observed due to Ag modification. These Ag- $\mathrm{ZnO}$ microneedle structures have been evaluated for their photocatalytic performance using methylene blue as model target contaminant and their activity was compared with the commercially available titania P25 photocatalyst. The photoactivity of all the Ag- $\mathrm{ZnO}$ microneedle structures was significantly higher than that of the commercially available $\mathrm{P} 25$ photocatalyst with the most active $\mathrm{Ag}-\mathrm{ZnO}$ material having a photocatalytic activity $\sim 1.4$ times greater than that of P25 titania.
\end{abstract}

Keywords Heavy Ag loading $\cdot \mathrm{Ag}-\mathrm{ZnO}$ microneedles $\cdot$ Surface plasmon $\cdot$ Electron density $\cdot$ Solar energy $\cdot$ Photocatalysis

\section{Introduction}

$\mathrm{ZnO}$ has been widely used as a photocatalyst in its pure form or after modification by the incorporation of foreign materials such as metals (Vaiano et al. 2018), non-metals (Kumari et al. 2019), and organics (Ansari et al. 2013). ZnO is a semiconductor with a large band gap of $3.37 \mathrm{eV}$. It is a suitable candidate in photocatalysis due to its ecofriendly nature, low cost, and high catalytic efficiency (Ong et al. 2018). When a photon energy of higher than or equal to its band gap energy is irradiated onto $\mathrm{ZnO}$, the electrons in the valance band (VB) jump to the conduction band $(\mathrm{CB})$ leading to the formation of

Responsible editor: Suresh Pillai

Electronic supplementary material The online version of this article (https://doi.org/10.1007/s11356-020-09433-5) contains supplementary material, which is available to authorized users.

Sanjay Gopal Ullattil

sanjaygopal.u@gmail.com

Ahmed Abdel-Wahab

ahmed.abdel-wahab@qatar.tamu.edu

1 Chemical Engineering Program, Texas A\&M University at Qatar, 23874, Education City, Doha, Qatar

2 Department of Nanoscience and Technology, University of Calicut, Malappuram, Kerala 673635, India electron-hole pairs (Ong et al. 2018). These electron-hole pairs can recombine within nanoseconds, which limits the photocatalytic efficiency of $\mathrm{ZnO}$ material. This unfavorable situation can be overcome by introducing a noble metalsemiconductor interface that can generate a Schottky barrier effect to inhibit the electron-hole recombination and thereby increase the photocatalytic efficiency (Yan et al. 2014).

Silver is one of the prominent candidates that can be used as dopant/modifier which generate $\mathrm{Ag}$-loaded $\mathrm{ZnO}$ nanostructures $(\mathrm{Ag}-\mathrm{ZnO})$ with a lower Fermi level $\left(E_{\mathrm{f}}\right)$ than the $\mathrm{CB}$ of $\mathrm{ZnO}$ (Ren et al. 2010). Thus, the photogenerated electron transfers to the $E_{\mathrm{f}}$ of $\mathrm{Ag}-\mathrm{ZnO}$ allowing $\mathrm{Ag}$ to act as a sink for electrons promote the interfacial charge transfer kinetics between $\mathrm{Ag}$ and $\mathrm{ZnO}$, and reduce the electron-hole recombination, which results in enhancing the photocatalytic efficiency. Also, Ag can originate surface plasmons and increase the absorption wavelength, thereby enhancing the visible light photocatalytic activity (Kavitha et al. 2019).

The morphology of the photocatalyst surface plays an important role in its photoactivity. We have previously developed a solution processing strategy for the synthesis of $\mathrm{ZnO}$ microrods (Ullattil et al. 2016) which has been adopted in this study for $\mathrm{Ag}$ doping $(10 \mathrm{~mol} \%)$ on $\mathrm{ZnO}$ to fabricate $\mathrm{Ag}-\mathrm{ZnO}$ microneedles at various high temperatures. $\mathrm{AgNO}_{3}$ that was used as the source of Ag has its ability to act as a morphological sharpener. The presented method is very suitable for industrial production of $\mathrm{Ag}-\mathrm{ZnO}$ microneedles as 
photocatalysts. An elongated prismatic growth can be favored by zinc precipitation using $\mathrm{NH}_{4} \mathrm{OH}$ and this has been welldocumented (Ullattil et al. 2016). The influence of zinc counterions on the shape evolution (Govender et al. 2004) and the $\mathrm{pH}$ dependence of $\mathrm{ZnO}$ and $\mathrm{Zn}(\mathrm{OH})_{2}$ formation are also available (Zhao et al. 2006). The present work was designed and planned to exploit the following features of an Ag-modified $\mathrm{ZnO}$ such as (1) $\mathrm{Zn}(\mathrm{OH})_{2}$ has been suggested as a prerequisite for the controlled growth of $\mathrm{ZnO}$ needles (Ullattil et al. 2016; McBride et al. 2003), (2) the lower Fermi level of Ag as compared with the $\mathrm{CB}$ of $\mathrm{ZnO}$ (Ren et al. 2010), and (3) strong ability of $\mathrm{Ag}$ to form a $\mathrm{Ag}-\mathrm{ZnO}$ interface and to generate a Schottky barrier for minimizing the $\mathrm{e}^{-}-\mathrm{h}^{+}$recombination (Yan et al. 2014). In addition, heavy loading of $\mathrm{AgNO}_{3}$ is employed for $\mathrm{Ag}$ incorporation into the $\mathrm{ZnO}$ crystal lattice rather than forming a surface linkage between them.

\section{Materials and methods}

\section{Materials}

Zinc nitrate hexahydrate $98 \%$ (Sigma-Aldrich), ammonium hydroxide (30\%, Merck), nitric acid (72\%, Merck), and silver nitrate, ACS reagent, $\geq 99 \%$ (Sigma-Aldrich) were purchased and were used as received. The dye used for photocatalytic study was methylene blue (Aldrich Chemicals) and was used without further purification. Deionized water was used in all the experiments.

\section{Preparation of Ag-modified ZnO}

In a typical experiment, zinc nitrate $(18.6 \mathrm{~g}, 0.25 \mathrm{M})$ was dissolved in $250 \mathrm{~mL}$ of water. The solution was stirred and ammonium hydroxide was slowly added drop by drop until $\mathrm{pH}$ reaches a value of 8 to ensure complete precipitation of white zinc hydroxide. After stirring for $2 \mathrm{~h}$, the so-formed zinc hydroxide solid was filtered using a Buchner apparatus. The precipitate was washed until it was free from ammonium and nitrate ions. The washed precipitate was dispersed in $300 \mathrm{~mL}$ of water and stirred continuously for $3 \mathrm{~h}$. At the same time, $10 \%$ nitric acid was added drop by drop to prevent aggregation of particles until the solution $\mathrm{pH}$ reached $\mathrm{pH} 6$ (Ullattil et al. 2016). For silver modification, $0.025 \mathrm{M}$ silver nitrate ( $1.061 \mathrm{~g}$ in $250 \mathrm{~mL}$ of water) was added to the above suspension and stirred continuously again for $6 \mathrm{~h}$. The suspension was allowed to dry at $100{ }^{\circ} \mathrm{C}$. The so-formed Ag-modified $\mathrm{ZnO}$ precursor $(\mathrm{AZ})$ was calcined at high temperatures 300 , 500 , and $700{ }^{\circ} \mathrm{C}$ for $2 \mathrm{~h}$ at a temperature ramp rate of $5{ }^{\circ} \mathrm{C}$ $\mathrm{min}^{-1}$ followed by heating at the highest temperature and is herein after termed as AZ3, AZ5, and AZ7 respectively.

\section{Characterization}

The crystallinity of Ag-modified $\mathrm{ZnO}$ powders was characterized by X-ray diffraction (XRD) technique with Miniflex 600 $X$-ray diffractometer in the diffraction angle range $2 \theta=20$ $80^{\circ}$ using $\mathrm{Cu} \mathrm{K} \alpha$ radiation. The average crystallite size " $\varphi$ " of the samples was estimated using Scherrer's equation (Eq. 1), by measuring the line broadening of (101) main intensity peak, where $\lambda$ is the wavelength of $\mathrm{Cu} \mathrm{K} \alpha$ radiation $(\lambda=$ $1.5401 \mathrm{~nm}), \beta$ is the full width at half-maximum, and $\theta$ is Bragg's angle (Cullity and Stock 2001). Surface morphologies were examined by using Hitachi-Su6600 field emission scanning electron microscope. The formation of $\mathrm{ZnO}$ was further confirmed by measuring Fourier transform infrared spectroscopy (FTIR) spectra of Ag-ZnO samples using Jasco-FT/IR-4100 spectrophotometer in the range of 4000 $400 \mathrm{~cm}^{-1}$. The Raman and photoluminescence spectra were performed using Thermo Fischer Raman spectrophotometer and Perkin Elmer LS 45 spectrophotometer. Photocatalysis of all the samples were studied using Xe lamp $(450 \mathrm{~W})$ as simulated solar light attached with Scientech power control. The distance from the light source to the solution was set to $18 \mathrm{~cm}$ and the intensity was measured using a Global solar power instrument and the measured intensity was $100 \mathrm{~mW} \mathrm{~cm}$. Both the optical measurements and photocatalytic studies were recorded with Jasco-V-550-UV/VIS spectrophotometer.

$\varphi=\frac{0.9 \lambda}{\beta \operatorname{Cos} \theta}$

\section{Photocatalysis}

The powdered sample $(0.1 \mathrm{~g})$ was dispersed in $50 \mathrm{~mL}$ of methylene blue dye solution of concentration $10^{-5} \mathrm{M}$ and stirred in the dark for $4 \mathrm{~h}$ to eliminate the possibility for adsorption during the photocatalytic reaction and thus maintaining the adsorption-desorption equilibrium. Then, the solution was irradiated under 1 sun with continuous stirring. Samples were collected in each $2 \mathrm{~min}$ and centrifuged for $5 \mathrm{~min}$ at $3000 \mathrm{rpm}$ to separate solid particles, followed by the absorption spectra measurements using a UV spectrophotometer.

\section{Results and discussion}

\section{Mechanism}

$\mathrm{Zn}(\mathrm{OH})_{2}$ is a prerequisite for the controlled growth of $\mathrm{ZnO}$ needles (McBride et al. 2003) and an elongated prismatic growth can be favored by $\mathrm{Zn}(\mathrm{OH})_{2}$ precipitation by additions of $\mathrm{NH}_{4} \mathrm{OH}$ to solutions containing $\mathrm{Zn}^{2+}$ which has also been reported (Ullattil et al). In this study, zinc nitrate was dissolved 
in water as $\mathrm{Zn}^{2+}$ and $\mathrm{NO}_{3}{ }^{-}$and $\mathrm{Zn}^{2+}$ ions precipitated as $\mathrm{Zn}(\mathrm{OH})_{2}$ after the addition of $\mathrm{NH}_{4} \mathrm{OH}$ base. The addition of $\mathrm{HNO}_{3}$ to $\mathrm{Zn}(\mathrm{OH})_{2}$-dispersed solution favored a $\mathrm{H}^{+}$ion templating around the precipitated $\mathrm{Zn}(\mathrm{OH})_{2}$ particles. The $\mathrm{pH}$ of the solution was adjusted to $\mathrm{pH} 6$. The $\mathrm{Ag}^{+}$as well as additional amounts of $\mathrm{NO}_{3}^{-}$was supplied upon the addition of $\mathrm{AgNO}_{3}$ to the solution of $\mathrm{pH}$ 6. According to the phase stability diagrams for the $\mathrm{ZnO}-\mathrm{H}_{2} \mathrm{O}$ and $\mathrm{Zn}(\mathrm{OH})_{2}-\mathrm{H}_{2} \mathrm{O}$ systems at $298 \mathrm{~K}$ and the thermodynamic data, the soluble species such as $\mathrm{Zn}^{2+}$ and $\mathrm{ZnOH}^{+}$are stable below pH 7 (Padmanabhan et al. 2009). These structures on to the oxygen-rich face of the Ag-incorporated $\mathrm{ZnO}$ nuclei occur to form microrod particles having an outermost $\mathrm{Zn}^{2+}$ layer at its one end. Such particles then grow to form the microneedles by a heterogeneous nucleation-assisted growth among the $\mathrm{Ag}$-incorporated $\mathrm{ZnO} / \mathrm{Zn}(\mathrm{OH})_{2}$ species. The consumption of more $\mathrm{NO}_{3}{ }^{-}$species upon $\mathrm{AgNO}_{3}$ addition and calcination at high temperatures results in the elimination of the growth barrier that in turn dictates the tapering of one end of the particles to form pointed tips, hence microneedles. These morphological evolutions of all the Ag-modified $\mathrm{ZnO}$ samples were followed using scanning electron microscopy (SEM), which led us to some interesting observations regarding the microneedle formation.

\section{Scanning electron microscopy}

The micrographs show that the precursor (AZ) has no specific morphology although it tends to form rod morphology even at $100{ }^{\circ} \mathrm{C}$ as shown in Fig. 1a. As the calcination temperature increased to $300{ }^{\circ} \mathrm{C}$ (AZ3), needles are also formed along with rods and thus the growth of microneedles is confirmed (Fig. 1b). While looking into $\mathrm{AZ5}$, uniform distribution of microneedles is observed (Fig. 1c) which could facilitate high degree of photocatalysis. The uniform distribution of microneedles has been destroyed at $700{ }^{\circ} \mathrm{C}$. As it can be seen in Fig. 1d, the SEM of AZ7, the pointed tips of the needles almost disappeared and tend to aggregate. This is due to the densification of $\mathrm{ZnO}$ normally observed at high temperatures (Mazaheri et al. 2008). $\mathrm{Ag}$ doping led the way to the formation of needle-like structures which are formed from $\mathrm{ZnO}$ microrods; i.e., $\mathrm{AgNO}_{3}$ is working as a sharpener of the microrods, and so in the present context, $\mathrm{AgNO}_{3}$ acts here as a "structural sharpener."

\section{X-ray diffraction}

The XRD patterns of AZ3, AZ5, and AZ7 are shown in Fig. 2, and they show good agreement with the hexagonal wurtzite $\mathrm{ZnO}$ (JCPDS file no. 36-1451) (Kuriakose et al. 2014). It is also noticed that, as the calcination temperature increases, remarkable peak shift is observed in the XRD pattern (inset of Fig. 2) which is a clear confirmation of the incorporation of $\mathrm{Ag}^{+}$into the crystal lattice of $\mathrm{ZnO}$ (Liu et al. 2017). The incorporation of $\mathrm{Ag}^{+}$ions into the $\mathrm{Zn}^{2+}$ sites is too difficult to achieve because of the large ionic radius difference between them (126 pm for $\mathrm{Ag}^{+}$and $74 \mathrm{pm}$ for $\mathrm{Zn}^{2+}$ ) (Ahmad et al. 2013). Since the ionic radius of $\mathrm{Ag}^{+}$is far greater than that of $\mathrm{Zn}^{2+}$, it can be confirmed that $\mathrm{Ag}^{+}$possessed the interstitial position of $\mathrm{ZnO}$ crystal lattice. The average crystallite sizes obtained from XRD were 42.7, 34.3, and $43.4 \mathrm{~nm}$ respectively for AZ3, AZ5, and AZ7. It is worth noting that the amorphous $\mathrm{ZnO}$ precursor approaches crystallinity even at $100{ }^{\circ} \mathrm{C}$ after $\mathrm{Ag}$ incorporation (Fig. S1) (Zhao et al. 2006). From this figure, it is clear that Ag doping has minimized the amorphous peaks which were present in bare $\mathrm{ZnO}$. Interestingly, the most
Fig. 1 SEM image of Ag-ZnO microneedles: a AZ, b AZ3, c $\mathrm{AZ5}$, and $\mathbf{d} \mathrm{AZ7}$ (scale bar is 1 $\mu \mathrm{m})$

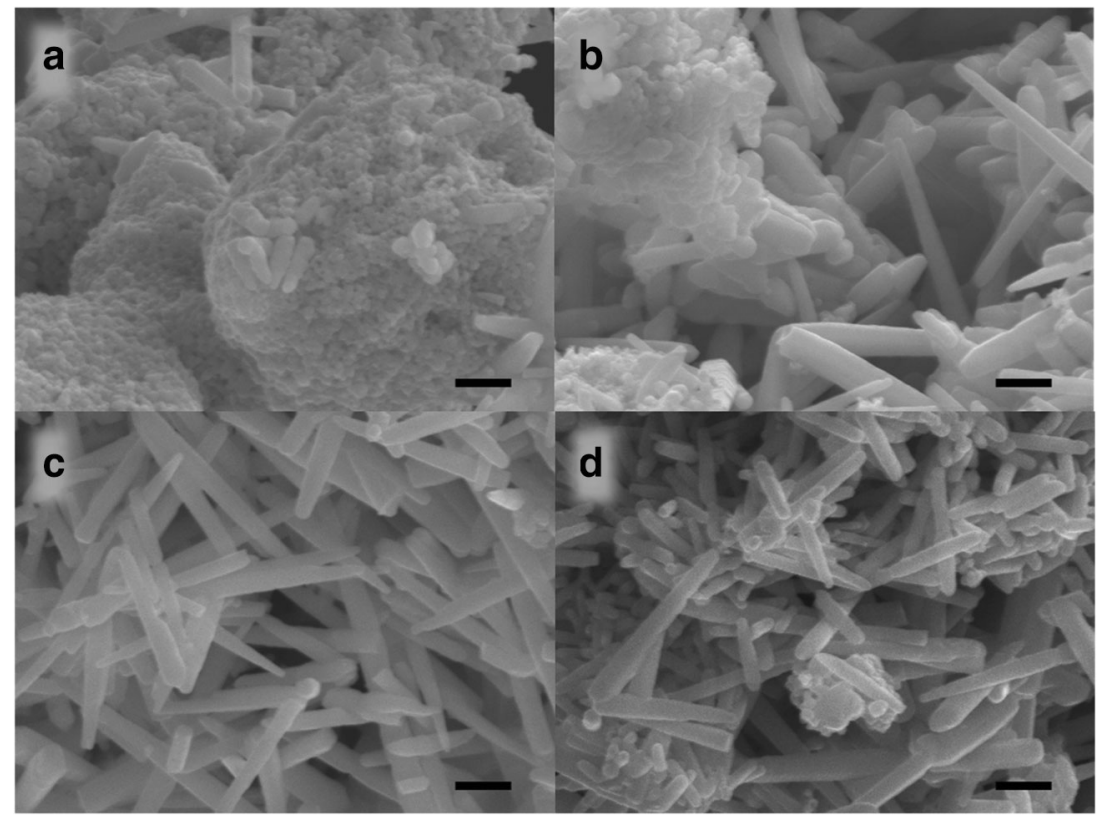


Fig. $2 \mathrm{XRD}$ of $\mathrm{Ag}-\mathrm{ZnO}$ microneedles-AZ3, AZ5, and AZ7, inset zoom out XRD peaks from 31 to $38^{\circ}$

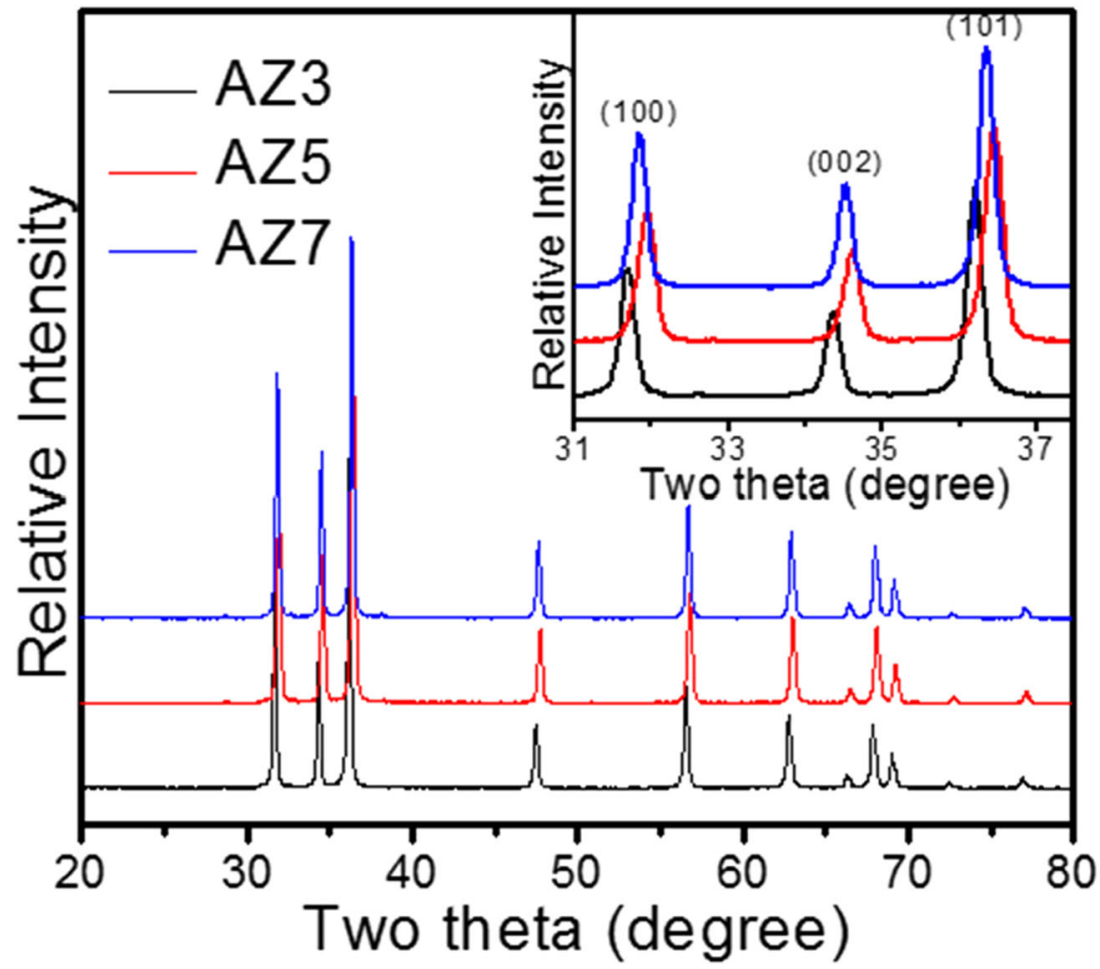

intense peak of $\mathrm{ZnO}$ precursor is a (300) peak which could be assigned to $\mathrm{Zn}(\mathrm{OH})_{2}$, whereas for the Ag- $\mathrm{ZnO}$ precursor, the most intense peak orientation is along the (100) plane which corresponds to the nanocrystalline $\mathrm{ZnO}$ (Pimentel et al. 2014). Thus, it can be confirmed that $\mathrm{AgNO}_{3}$ is not only a useful dopant and structural sharpener but also a phase purifier.

\section{Fourier transform infrared spectroscopy}

FTIR spectra were recorded to further confirm the formation of Ag-modified ZnO. From FTIR studies (Fig. 3), the broad band at $\sim 513 \mathrm{~cm}^{-1}$ is attributed to $\mathrm{Zn}-\mathrm{O}$ stretching vibration (Saravanan et al. 2011). The peaks at 3420 and $1633 \mathrm{~cm}^{-1}$ correspond to the stretching and bending vibrations of surfaceadsorbed $-\mathrm{OH}$ and water molecules (Moussawi and Patra 2016). Even though $\mathrm{Ag}^{+}$ions have been incorporated into the crystal lattice of $\mathrm{ZnO}$, no peaks are observed as characteristics of direct bonding between $\mathrm{Zn}$ and $\mathrm{Ag}$. Thus, it is argued that the $\mathrm{Ag}^{+}$ions that have been incorporated into the lattice sites possess the interstitial space of the $\mathrm{ZnO}$ structure. For all the samples, a band at $1017 \mathrm{~cm}^{-1}$ is present, which is ascribed to the threshold frequency band representing the activation energy for electronic conduction which is beneficial for the high photocatalytic activity (Jimenez-Gonzalez et al. 1998). This threshold energy band that was dominant in AZ5 also enhances the photocatalytic efficiency of AZ5 as compared with other samples, which will be discussed in detail under the Photocatalysis section. A high intense peak at $\sim 2354 \mathrm{~cm}^{-1}$ is due to surface-adsorbed $-\mathrm{CO}_{2}$ molecules (Saber et al. 2017) and as a consequence, the peaks at 2922 and $2852 \mathrm{~cm}^{-1}$ can be attributed to $\mathrm{C}-\mathrm{OH}$ peaks originated due to the interaction of $\mathrm{CO}_{2}$ with weakly adsorbed $-\mathrm{OH}$ groups (Saber et al. 2017). The E1(LO) mode of $\mathrm{ZnO}$ microneedles has also been shown in Fig. S2 and the band positions were found around 512 $\mathrm{cm}^{-1}$. According to previous report of Zheng et al., the E1(LO) mode should shift with the change in the density of oxygen vacancies (Zheng et al. 2007). In addition, the precursor (AZ) has an E1(LO) mode of vibration at $\sim 554 \mathrm{~cm}^{-1}$ whereas for all the other samples, they have bands at lower wave number at $\sim 512 \mathrm{~cm}^{-1}$ indicating the low amount of oxygen vacancy defects in all the samples except AZ. In other words, the defect states are more in the $\mathrm{ZnO}$ precursor and the defect states are highly reduced when calcined at $300-700{ }^{\circ} \mathrm{C}$.

\section{UV-visible absorption spectra and Tauc plots}

The absorption spectra of Ag-ZnO samples are presented in Fig. 4a. As the figure indicates, with the increase in temperature, surface plasmon resonance peak shift occurs. Generally, surface plasmon peaks for $\mathrm{Ag}$ are observed at the wavelength range of 430-460 nm. Here, for Ag- $\mathrm{ZnO}$ precursor, the plasmon peak is observed at $463 \mathrm{~nm}$ and as the temperature increased to $300^{\circ} \mathrm{C}$, the peak red shifted to $466 \mathrm{~cm}^{-1}$. Interestingly at $500{ }^{\circ} \mathrm{C}$, the surface plasmon peak is red shifted to a large extent and the value is at $544 \mathrm{~cm}^{-1}$. But as the temperature reached to 700 ${ }^{\circ} \mathrm{C}$, a blue shift in plasmon peak was observed having a value of $535 \mathrm{~cm}^{-1}$. The plasmon absorption $\left(\lambda_{\mathrm{p}}\right)$ of silver can be represented as Eq. 2 (Chen et al. 2011) 
Fig. 3 FTIR spectra of Ag-ZnO micro needles-AZ3, AZ5, and AZ7

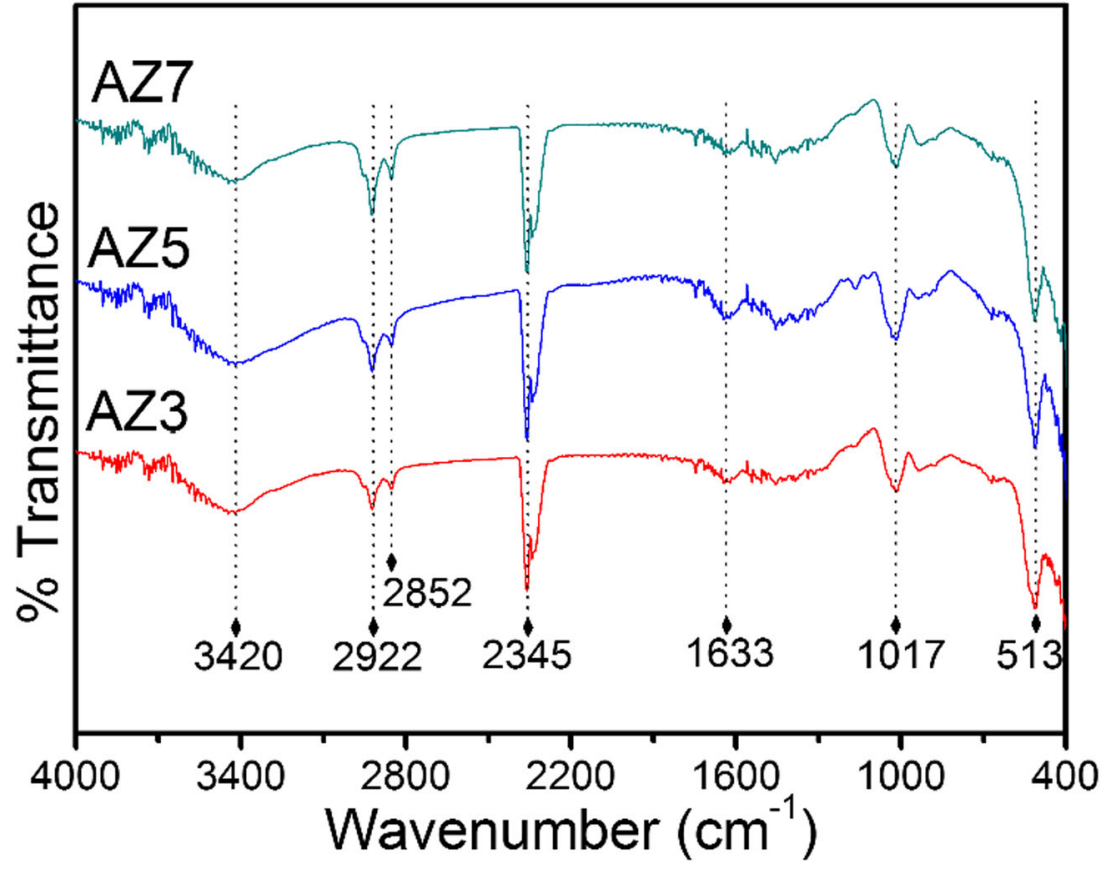

Tauc plot of all the calcined samples are also shown in Fig. 4b, where the band gap energy slightly decreased as the calcination temperature increased and the values observed are 3.04, 3.00, and $2.95 \mathrm{eV}$ for AZ3, AZ5, and AZ7 respectively.

\section{Raman spectroscopy}

To further confirm the crystallinity of the synthesized solids, Raman spectroscopy was used and the spectra are displayed in Fig. 5. All the Ag-ZnO samples show a sharp peak at $\sim 440 \mathrm{~cm}^{-1}$ corresponding to the $\mathrm{E}_{2}$ (high) mode of the Raman active mode which is the main characteristic of wurtzite hexagonal phase of $\mathrm{ZnO}$ (Ngo-Duc et al. 2012). Generally, a peak of $\mathrm{E}_{1}$ (LO) mode around $580 \mathrm{~cm}^{-1}$ is observed for $\mathrm{ZnO}$ having oxygen vacancy defect states and no such peak is visible for any of the samples, which reveals the low concentration of oxygen vacancy states

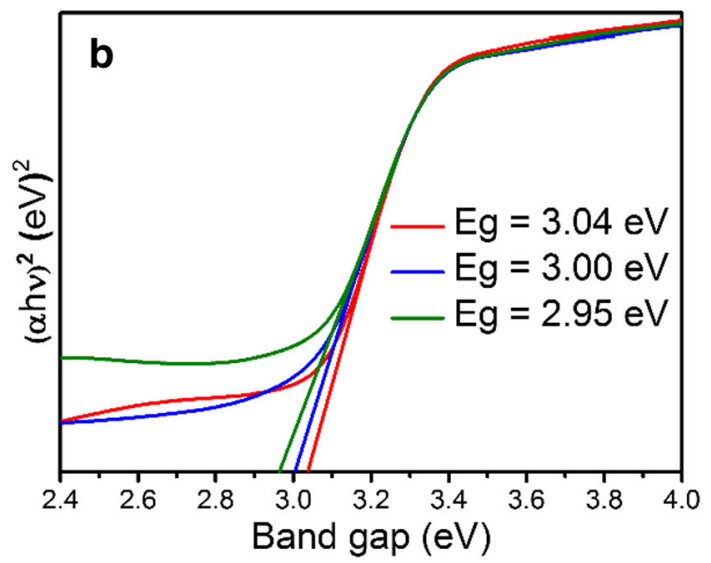

Fig. 4 a UV-visible spectra of Ag-ZnO needles-AZ3, AZ5, and AZ7 — and b Tauc plot of Ag-ZnO microneedles-AZ3, AZ5, and AZ7 
and higher crystalline nature of the materials (KhosraviGandomani et al. 2014). This finding is in agreement with the E1 (LO) mode of the samples obtained from FTIR. In addition, AZ3 and AZ5 shows the peak at $\sim 331 \mathrm{~cm}^{-1}$ ascribed to $E_{2 \mathrm{H}^{-}} E_{2 \mathrm{~L}}$ (multi phonon process mode) which is absent in AZ7 indicating that both of these samples show single crystalline nature but AZ7 does not (Umar and Hahn 2006).

\section{Photoluminescence spectroscopy}

The photoluminescence spectra of the Ag-doped samples are shown in Fig. 6a. In the inset of the figure, the deconvoluted spectra of the photoluminescence spectroscopy (PL) in the range $450-650 \mathrm{~nm}$ fitted using the Gaussian fit method (to differentiate the peaks) are given in order to differentiate the deep level emissions of the doped samples. The spectra generally indicate lower defect state after doping. In-depth investigation of the spectra reveals that the band edge emission is observed at $394 \mathrm{~nm}$ in the UV region and is almost equivalent to $3.14 \mathrm{eV}$. The convolution resulted in the peaks obtained at $483,531,574$, 596 , and $630 \mathrm{~nm}$. The peak at $483(2.56 \mathrm{eV})$ is assigned to electron transition from zinc vacancy level observed at $0.28 \mathrm{eV}$ from conduction band level to single ionized zinc vacancies $(2.84 \mathrm{eV})$ resulting a blue emission spectra (Lin et al. 2001). A green emission is observed at $531(2.34 \mathrm{eV}) \mathrm{nm}$ due to electron transition from the conduction band to the oxygen ion vacancies (Vo) situated at $\sim 0.8 \mathrm{eV}$ from the valence band. As oxygen ion vacancies are lower, the peak is almost negligible in the spectrum (Amiruddin and Kumar 2014a, b). The peak at 574 $(2.16 \mathrm{eV})$ and $596(2.08 \mathrm{eV})$ resulting in a yellow and orange emission respectively is probably due to transition of electrons from the conduction band and zinc ion vacancy to the oxygen interstitials (Oi) located at $\sim 1 \mathrm{eV}$ (Amiruddin and Kumar 2014a, b). Red emissions at $630 \mathrm{~nm}$ are negligibly small and

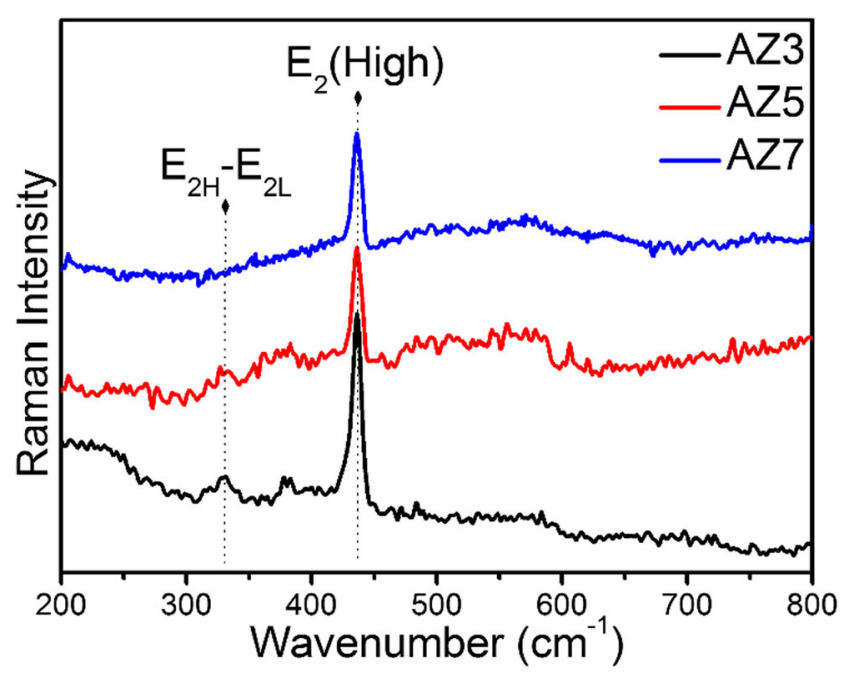

Fig. 5 Raman spectra of Ag-ZnO microneedles-AZ3, AZ5, and AZ7 hence are almost invisible indicating deep level emissions are almost nil. The overall PL spectrum indicates minimum defect states in the zinc oxide after doping with silver. A schematic representation of the emission is given in Fig. 6b. These results regarding the defect states are in good agreement with the results obtained from E1 (LO) mode of FTIR and the Raman spectra.

\section{Photocatalysis}

Methylene blue (MB) is adopted as the representative organic pollutant to evaluate the photocatalytic performance of $\mathrm{Ag}-\mathrm{ZnO}$ microneedles. Commercially available photocatalyst P25 titania has also been evaluated and thus used as a reference photocatalyst. The photocatalytic activities of the as-prepared Ag-modified sample at different temperatures and P25 are shown in Fig. 7. $C_{0}$ and $C$ are the initial concentration after reaching the adsorption equilibrium and the measured concentration at the corresponding reaction time of $\mathrm{MB}$ respectively. As seen in Fig. 7, the degradation of $\mathrm{MB}$ over the Ag- $\mathrm{ZnO}$ microneedles exhibits higher photocatalytic activity compared with commercially available P25 titania photocatalyst. The degradation of MB over P25, AZ3, AZ5, and AZ7 catalysts was monitored over $8 \mathrm{~min}$ and the maximum photoactivity was observed for AZ5. The degradation rate percentage $(x)$ in Eq. 3 was $84.8,92.1$, and 88.2 for AZ3, AZ5, and AZ7 respectively, whereas the photodegradation rate percentage of P25 was 67.2 after $8 \mathrm{~min}$. The order of photoactivity was AZ5 > AZ7 > AZ3 > P25 and it can be seen that all the photocatalytic processes here followed the first order kinetics (Fig. S4).

$x=\frac{C_{0}-C}{C_{0}} \times 100$

The Fermi level originated due to $\mathrm{Ag}$ modification has restrained the electron-hole recombination during photocatalysis and thus, Ag acts as a sink of electrons. Here, for all the samples, the activation energy for electronic conduction is high which is evident from FTIR. The corresponding band is slightly higher in intensity for AZ5 and hence, high photocatalytic activity is observed. In addition to this, the microneedle morphology, surface plasmon effect, and single crystalline nature were collectively beneficial for the high photoactivity of all the samples. The observed electron density $(N)$ was highest for AZ3 and the order can be represented as $N_{\mathrm{AZ} 5}<N_{\mathrm{AZ7} 7}<N_{\mathrm{AZ3} 3}$. Obviously, the order of effective mass of free electrons would be the reverse and can be represented as $N_{\mathrm{AZ} 5}>N_{\mathrm{AZ7} 7}>N_{\mathrm{AZZ}}$. Generally, the higher effective mass of free electrons leads to reduced photocatalytic performance. However, here, the maximum efficiency is observed for AZ5 which has the maximum effective mass of electrons. If a large difference between the effective masses of holes $\left(m_{\mathrm{h}+}\right)$ and electrons $\left(m_{\mathrm{e}-)}\right.$ occur, the condition may be reversed. The 


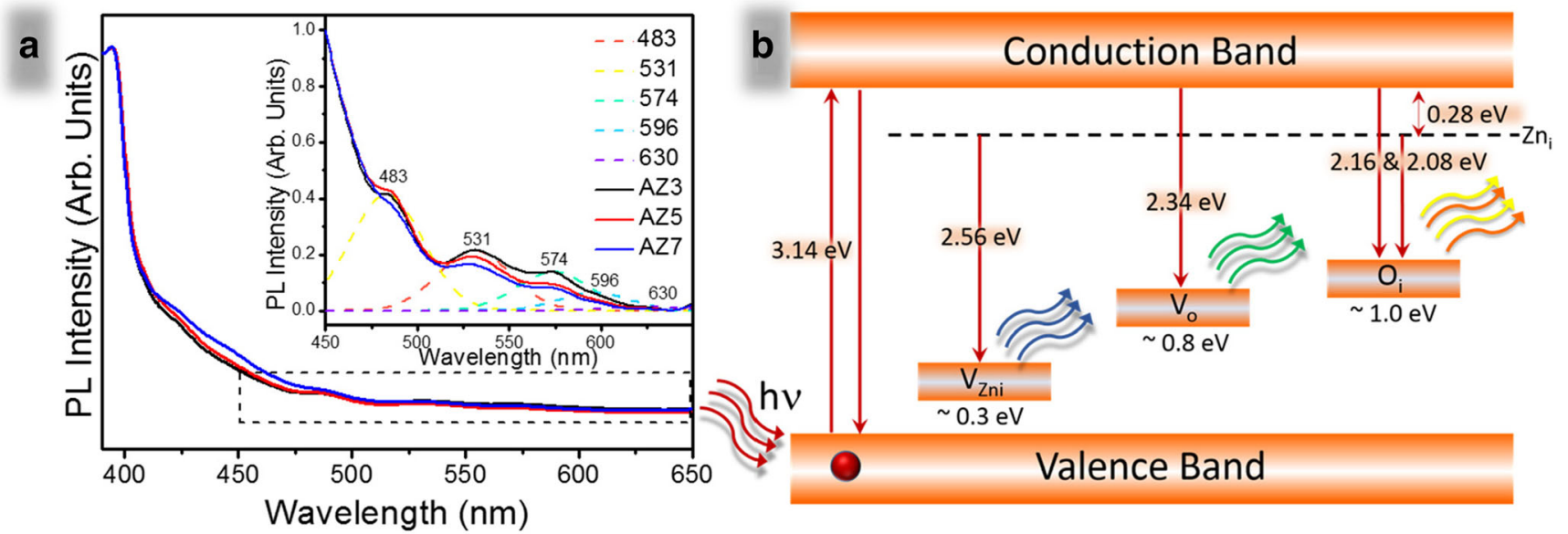

Fig. 6 a PL spectra of Ag-ZnO microneedles - AZ3, AZ5, and AZ7 — and $\mathbf{b}$ schematic representation of emission of photons from various defect states

relative ratio of effective masses $(D)$ can be explained as Eq. 4 (Faraji et al. 2015)

$D=m_{\mathrm{h}+} / m_{\mathrm{e}^{-}}$

If the $D$ value is higher, the electron mobility would increase and thus, the recombination of the photoinduced charges can be restrained (Opoku et al. 2017). So, it is expected that the effective mass of holes is greater for all the samples and therefore, the order of the relative ratio of effective masses $(D)$ as well as the photocatalytic efficiency follows the order AZ5 > AZ7 > AZ3.

\section{Conclusions}

A modified solution processing strategy has been developed for the synthesis of Ag-modified $\mathrm{ZnO}$ microneedles with minimized defect states. The Ag-modified $\mathrm{ZnO}$ precursor was calcined at 300,500 , and $700{ }^{\circ} \mathrm{C}$. The facile incorporation of $\mathrm{Ag}^{+}$into the

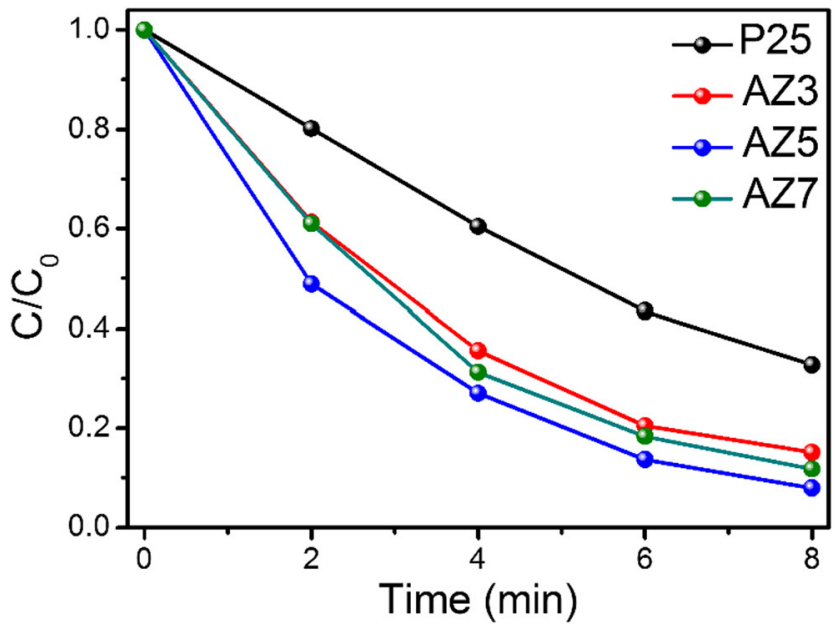

Fig. 7 Photodegradation kinetics of MB under 1 sun using P25, AZ3, AZ5, and AZ7
$\mathrm{ZnO}$ led the way to minimization of the amorphous peaks that in bare $\mathrm{ZnO}$ precursor synthesized at $100{ }^{\circ} \mathrm{C}$. In addition to that, the morphology of all the Ag-modified $\mathrm{ZnO}$ samples is microneedles. Absorption spectra proved the high visible light absorption of Agmodified $\mathrm{ZnO}$ samples, which has extended the wavelength cutoff toward higher wavelength visible region. The "sharpening effect" of $\mathrm{AgNO}_{3}$ was confirmed by SEM analysis, which has resulted in the morphological conversion of microrods to needles. These materials were employed as photocatalysts for the photodegradation of methylene blue under 1 sun solar illumination. The photoactivity followed the order of AZ5 $>$ AZ7 $>$ AZ3 $>$ P25, where the percentage of photodegradation for the most active performer AZ5 and P25 was 92.1 and 67.2 after 8 min of 1 sun solar illumination. It is expected that the efficiency of these materials can be exploited in photocatalytic water splitting and other solar energy applications.

Acknowledgments Open Access funding provided by the Qatar National Library. Authors are thankful to Dr. Pradeepan Periyat, Assistant Professor, University of Calicut, for providing Raman and photoluminescence characterization facilities.

Funding information This research was supported by a grant from Texas A\&M University at Qatar under its Responsive Research Seed Grants (RRSG) Program.

Open Access This article is licensed under a Creative Commons Attribution 4.0 International License, which permits use, sharing, adaptation, distribution and reproduction in any medium or format, as long as you give appropriate credit to the original author(s) and the source, provide a link to the Creative Commons licence, and indicate if changes were made. The images or other third party material in this article are included in the article's Creative Commons licence, unless indicated otherwise in a credit line to the material. If material is not included in the article's Creative Commons licence and your intended use is not permitted by statutory regulation or exceeds the permitted use, you will need to obtain permission directly from the copyright holder. To view a copy of this licence, visit http://creativecommons.org/licenses/by/4.0/. 


\section{References}

Ahmad M, Ahmed E, Hong ZL, Khalid NR, Ahmed W, Elhissi A (2013) Graphene- $\mathrm{Ag} / \mathrm{ZnO}$ nanocomposites as high performance photocatalysts under visible light irradiation. J Alloys Compnd 577:717-727. https://doi.org/10.1016/j.jallcom.2013.06.137

Amiruddin R, Kumar MS (2014a) Enhanced visible emission from vertically aligned $\mathrm{ZnO}$ nanostructures by aqueous chemical growth process. J Lumin 155:149-155. https://doi.org/10.1016/j.jlumin. 2014.06.038

Amiruddin R, Kumar MS (2014b) Epitaxial growth of vertically aligned highly conducting $\mathrm{ZnO}$ nanowires by modified aqueous chemical growth process. Ceram Int 40:11283-11290. https://doi.org/10. 1016/j.ceramint.2014.03.154

Ansari SA, Khan MM, Ansari MO, Lee J, Cho MH (2013) Biogenic synthesis, photocatalytic, and photoelectrochemical performance of $\mathrm{Ag}-\mathrm{ZnO}$ nanocomposite. J Phys Chem C 117:27023-27030. https://doi.org/10.1021/jp410063p

Chen C, Zheng Y, Zhan Y, Lin X, Zheng Q, Wei K (2011) Enhanced Raman scattering and photocatalytic activity of $\mathrm{Ag} / \mathrm{ZnO}$ heterojunction nanocrystals. Dalton Trans 40:9566-9570. https:// doi.org/10.1039/C1DT10799B

Cullity BD, Stock SR (2001) Elements of X-ray diffraction. Prentice hall, New Jersey

Faraji M, Sabzali M, Yousefzadeh S, Sarikhani N, Ziashahabi A, Zirak M, Moshfegh AZ (2015) Band engineering and charge separation in the $\mathrm{Mo}_{1-\mathrm{x}} \mathrm{W}_{\mathrm{x}} \mathrm{S}_{2} / \mathrm{TiO}_{2}$ heterostructure by alloying: first principle prediction. RSC Adv 5:28460-28466. https://doi.org/10.1039/ C5RA00330J

Govender K, Boyle DS, Kenway PB, O’Brien P (2004) Understanding the factors that govern the deposition and morphology of thin films of $\mathrm{ZnO}$ from aqueous solution. J Mater Chem 14:2575-2591. https://doi.org/10.1039/B404784B

Jimenez-Gonzalez AE, Urueta JA, Suarez-Parra R (1998) Optical and electrical characteristics of aluminum-doped $\mathrm{ZnO}$ thin films prepared by solgel technique. J Cryst Growth 192:430-438. https:// doi.org/10.1016/S0022-0248(98)00422-9

Kavitha R, Girish Kumar S, Sushma CG (2019) Plasmonic Ag-ZnO: charge carrier mechanisms and photocatalytic applications. Photocatalytic Functional Materials for Environmental Remediation. pp. 191-214.

Khosravi-Gandomani S, Yousefi R, Jamali-Sheini F, Huang NM (2014) Optical and electrical properties of p-type Ag-doped $\mathrm{ZnO}$ nanostructures. Ceram Int 40:7957-7963. https://doi.org/10.1016/j.ceramint. 2013.12.145

Kumari V, Mittal A, Jindal J, Yadav S, Kumar N (2019) S-, N-and Cdoped $\mathrm{ZnO}$ as semiconductor photocatalysts: a review. Front Mater Sci 13:1-22. https://doi.org/10.1007/s11706-019-0453-4

Kuriakose S, Choudhary V, Satpati B, Mohapatra S (2014) Facile synthesis of $\mathrm{Ag}-\mathrm{ZnO}$ hybrid nanospindles for highly efficient photocatalytic degradation of methyl orange. Phys Chem Chem Phys 16: 17560-17568. https://doi.org/10.1039/C4CP02228A

Lin B, Fu Z, Jia Y (2001) Green luminescent center in undoped zinc oxide films deposited on silicon substrates. Appl Phys Lett 79:943-945. https://doi.org/10.1063/1.1394173

Liu FC, Li JY, Chen TH, Chang CH, Lee CT, Hsiao WH, Liu DS (2017) Effect of silver dopants on the $\mathrm{ZnO}$ thin films prepared by a radio frequency magnetron co-sputtering system. Materials 10:797. https://doi.org/10.3390/ma10070797

Mazaheri M, Zahedi AM, Sadrnezhaad SK (2008) Two-step sintering of nanocrystalline $\mathrm{ZnO}$ compacts: effect of temperature on densification and grain growth. J Am Ceram Soc 91:56-63. https://doi.org/ 10.1111/j.1551-2916.2007.02029.x
McBride RA, Kelly JM, McCormack DE (2003) Growth of well-defined $\mathrm{ZnO}$ microparticles by hydroxide ion hydrolysis of zinc salts. J Mater Chem 13:1196-1201. https://doi.org/10.1039/B211723C

Moussawi RN, Patra D (2016) Modification of nanostructured ZnO surfaces with curcumin: fluorescence-based sensing for arsenic and improving arsenic removal by ZnO. RSC Adv 6:17256-17268. https://doi.org/10.1039/C5RA20221C

Ngo-Duc T, Singh K, Meyyappan M, Oye MM (2012) Vertical ZnO nanowire growth on metal substrates. Nanotechnol 23:194015. https://doi.org/10.1088/0957-4484/23/19/194015

Ong CB, Ng LY, Mohammad AW (2018) A review of ZnO nanoparticles as solar photocatalysts: synthesis, mechanisms and applications. Renew Sust Energ Rev 81:536-551. https://doi.org/10.1016/j.rser. 2017.08.020

Opoku F, Govender KK, van Sittert CG, Govender PP (2017) Understanding the mechanism of enhanced charge separation and visible light atalytic activity of modified wurtzite $\mathrm{ZnO}$ with nanoclusters of $\mathrm{ZnS}$ and graphene oxide: from a hybrid density functional study. New J Chem 41:8140-8155. https://doi.org/10. 1039/C7NJ01942D

Padmanabhan SC, Ledwith D, Pillai SC, McCormack DE, Kelly JM (2009) Microwave-assisted synthesis of $\mathrm{ZnO}$ micro-javelins. J Mater Chem 19:9250-9259. https://doi.org/10.1039/B912537J

Pimentel A, Nunes D, Duarte P, Rodrigues J, Costa FM, Monteiro T, Martins R, Fortunato E (2014) Synthesis of long ZnO nanorods under microwave irradiation or conventional heating. J Phys Chem C 118:14629-14639. https://doi.org/10.1021/jp5027509

Ren C, Yang B, Wu M, Xu J, Fu Z, Guo T, Zhao Y, Zhu C (2010) Synthesis of $\mathrm{Ag} / \mathrm{ZnO}$ nanorods array with enhanced photocatalytic performance. J Hazard Mater 182:123-129. https://doi.org/10.1016/ j.jhazmat.2010.05.141

Saber H, Alwaleed EA, Ebnalwaled KA, Sayed A, Salem W (2017) Efficacy of silver nanoparticles mediated by Jania rubens and Sargassum dentifolium macroalgae; Characterization and biomedical applications. Egypt J Basic Appl Sci 4:249-255. https://doi.org/ 10.1016/j.ejbas.2017.10.006

Saravanan R, Shankar H, Prakash T, Narayanan V, Stephen A (2011) $\mathrm{ZnO} / \mathrm{CdO}$ composite nanorods for photocatalytic degradation of methylene blue under visible light. Mater Chem Phys 125:277280. https://doi.org/10.1016/j.matchemphys.2010.09.030

Ullattil SG, Periyat P, Naufal B, Lazar MA (2016) Self-doped ZnO microrods-high temperature stable oxygen deficient platforms for solar photocatalysis. Ind Eng Chem Res 55:6413-6421. https:// doi.org/10.1021/acs.iecr.6b01030

Umar A, Hahn YB (2006) Aligned hexagonal coaxial-shaped ZnO nanocolumns on steel alloy by thermal evaporation. Appl Phys Lett 88:173120. https://doi.org/10.1063/1.2200472

Vaiano V, Matarangolo M, Murcia JJ, Rojas H, Navío JA, Hidalgo MC (2018) Enhanced photocatalytic removal of phenol from aqueous solutions using $\mathrm{ZnO}$ modified with Ag. Appl Catal B Environ 225:197-206. https://doi.org/10.1016/j.apcatb.2017.11.075

Yan F, Wang Y, Zhang J, Lin Z, Zheng J, Huang F (2014) Schottky or ohmic metal-semiconductor contact: influence on photocatalytic efficiency of $\mathrm{Ag} / \mathrm{ZnO}$ and $\mathrm{Pt} / \mathrm{ZnO}$ model systems. ChemSusChem.7: 101-104.

Zhao J, Jin ZG, Liu XX, Liu ZF (2006) Growth and morphology of ZnO nanorods prepared from $\mathrm{Zn}\left(\mathrm{NO}_{3}\right)_{2} / \mathrm{NaOH}$ solutions. J Eur Ceram Soc 26:3745-3752. https://doi.org/10.1016/j.jeurceramsoc.2006.01.006

Zheng Y, Zheng L, Zhan Y, Lin X, Zheng Q, Wei K (2007) Ag/ZnO heterostructure nanocrystals: synthesis, characterization, and photocatalysis. Inorg Chem 46:6980-6986. https://doi.org/10. 1021/ic700688f

Publisher's note Springer Nature remains neutral with regard to jurisdictional claims in published maps and institutional affiliations. 